\title{
The General Methodology of Analytical Environmental Control
}

\author{
DOI: $10.1134 / \mathrm{S} 1061934810030019$
}

The main problem of environmental analysis and control is, of course, the detection, identification, and quantitative determination of hazardous substances of anthropogenic origin. However, the problem of the control of natural components, for example, carbon dioxide and ozone in ambient air or dissolved oxygen in waters, is still being important.

The control and monitoring of environmental objects in terms of the determination of their chemical composition faces great difficulties. Some of these difficulties are due to the specific features of the environmental objects, for example, the diversity of their natural composition and their lability, i.e., time variability. Other difficulties originate from the magnitude of the problems and the volume of the necessary work. In fact, the number of the substances, whose concentration should evidently be controlled is growing. It is known that maximum permissible concentrations (MPCs) in natural waters are prescribed for approximately one and a half thousand substances. The number of required analyses even for the most widespread pollutants is being increased. Even new types of objects appear for which control is necessary. All this is good; it is indicative of the care and duty of society and government regarding the cleanliness of the environment and the improvement of the quality of life, but the duties of analysts and control services in the world should multiply significantly.

The situation is aggravated because the general methodology of chemical analysis and environmental control used nowadays does not permit solving complicated tasks. For example, for all 1500 substances normalized in waters, one must have reliable determination procedures. Let us suppose that these have been developed. However, none of the control laboratories can determine these substances simultaneously, if only because of the enormous efforts required. Of course, one understands that, in actual practice, all these substances should not be determined simultaneously, but this is quite another problem.

What do we actually have? Good domestic laboratories perform analyses for 100 to 150 indexes, average laboratories, for 20 to 30 indexes, and some of them for an even smaller number.

The way to resolve this is, probably, by modifying the methodology itself and of the general approaches to analysis and control.

The concept of environmental control in terms of the determination of its chemical composition can include several items; I will consider six of them. I would like to note that these items are closely related to one another.

Two-step analysis. The first item can be the provision of routine two-step (or even three-step) analysis with sample screening at the first step.

Screening makes the control much simpler and easier. At this first step, samples, for which negative results were obtained by a semiquantitative, simplified assessment of the presence or absence of the required substances, are rejected. The negative result, i.e., data on the absence of the required substances or, more precisely, about their possible presence in amounts smaller than the specified concentration limit, is considered definitive, and these samples are not considered further. Samples, for which a positive result was obtained, are transferred to the second step of analysis, where more precise methods are used.

At the first step, inexpensive and simple methods are convenient, because the task of this step is to ensure the mass scale and rapidity of assessments.

Generalized indexes. The second important item of the methodology can be the much wider use of generalized, integral indexes in comparison to the present time. In this way, one partially evades the problem of the most laborious component-by-component analysis. Generalized indexes are particularly valuable at the first step of control.

It is important that research into the search for new generalized indexes, their testing, and inserting into the system of multistep analysis will be organized.

In future, some widely known, but imperfect indexes, such as, for example, biological oxygen demand, BOD, will be, probably, excluded from use, and others (chemical oxygen demand, total organic carbon, tests for total heavy metals, etc.) will be sophisticated.

Special attention should be given to the development and construction of biotests, first of all, for water. These should be rapid, inexpensive, and based on the use of available, long-stored, and easily transported organisms. Biotests should be performed in field conditions, i.e., in simple conditions. The accepted rules also require that they must be certified. Unfortunately, the domestic standard base of biotests is relatively poor.

Biotests are used at the first step of control.

Pattern recognition. One more way to avoid component-by-component analysis (this time, almost 
completely) is the use of an approach, named pattern recognition. The recognition of the general image of a test sample object using, first of all, an electronic tongue or an electronic nose, has not been used in the practice of environmental analysis. However, with the expansion of commercial facilities of the specified type, this technique is believed to be used in the specified field. For example, an electronic tongue will evidently indicate changes in the composition of water.

In some cases, these systems can be used also for controlling pollution.

Shift of analysis from laboratory to the field. An obvious and in-force item of the concept under consideration is the shift of chemical analysis from stationary laboratories to the place of the test object, to the field.

The advantages of extralaboratory analysis are obvious. It saves time and resources for the preservation and transportation of samples. Samples can be analyzed in the real-time mode. Field analysis employs simpler and cheaper means than the laboratory one; therefore, the requirements for analysts are reduced. More importantly, there are objects that are difficult to analyze in the laboratory or whose analysis in the laboratory makes no sense. This is true for labile samples and emergency situations, including those of natural origin.

It is useful to store control samples for periodic tests in laboratories, particularly as some of the test means can easily be used for this purpose, for example, as tablets of polyurethane foams with analytes chemisorbed on them.

It is evident that the most complex analyses of the most complicate objects cannot be performed in the field.

Mass use of transportable analytical means, including simplest ones. The development and wide use of transportable analytical means favors the provision of routine analyses and their availability, in particular in field.

In principle, the arsenal of transportable means includes mobile laboratories on cars, boats, railway coaches, helicopters, and planes. Next, these are portable instruments, including pocket ones; chemical and biochemical test kits, test systems, and also, mainly in future, systems of chemical sensors. Among these facilities, mobile laboratories are, of course, palliative means, intermediate between stationary laboratories and systems of portable devices.

Nauka Publishing is going to issue a collective monograph Vnelaboratornyi Khimicheskii Analiz (Extralaboratory Chemical Analysis), in which great attention is paid to transportable analytical tools.

Automation of analysis. An essential requirement for the implementation of routine analyses is evidently their automation. One of the versions is the creation of stations and posts automatically working in a continuous mode and monitoring the composition of air and water. Thus, thirty-seven stations of air analysis operate in Moscow; they determine the concentration of $\mathrm{CO}, \mathrm{SO}_{2}, \mathrm{NH}_{3}$, and some organic substances. However, the most toxic organic compounds cannot be determined by this method.

Another version is the automation of routine laboratory analyses, first of all, of water. In particular, much has been done in using (more in the development) of such methods, based on flow-injection analysis and its analogues. This is, undoubtedly, a promising way. We should not forget that many up-to-date instruments are automated to a significant degree.

Generally speaking, there is one more point in the general methodology, organizational. In Russia, in spite of the decision approved long ago that a united state system of environmental monitoring (USSEM) must be organized, we still have not came to the harmonization of the requirements, approaches, and methodological basis between different ministries, the Ministry for the Protection of the Environment and Natural Resources, the Federal Hydrometereology and Environmental Monitoring Service, etc.

Yu. A. Zolotov 